\title{
Clinical Study \\ Decrease of Total Antioxidative Capacity in Developed Low Cardiac Output Syndrome
}

\author{
Alper Sami Kunt ${ }^{1}$ and Mehmet Halit Andac ${ }^{2}$ \\ ${ }^{1}$ Özel Yaşam Hastanesi Life Hospital, Antalya, Turkey \\ ${ }^{2}$ Özel Çekirge Kalp ve Artimi Hastanesi, Bursa, Turkey \\ Correspondence should be addressed to Alper Sami Kunt, dralper@msn.com
}

Received 23 June 2012; Accepted 14 October 2012

Academic Editor: Manikandan Panchatcharam

Copyright (C) 2012 A. S. Kunt and M. H. Andac. This is an open access article distributed under the Creative Commons Attribution License, which permits unrestricted use, distribution, and reproduction in any medium, provided the original work is properly cited.

\begin{abstract}
Background. It has been known that cardiac surgery induces an oxidative stress. The persistent oxidative stress during reperfusion may lead to depressed myocardial function resulting in low cardiac output syndrome (LCOS) necessitating inotropic or intraaortic balloon counterpulsation support. Total antioxidant capacity (TAC) is a measurement of oxidative stress in tissues. The purpose of this study was to examine the TAC differences during coronary artery bypass graft (CABG) operation in patients who have developed LCOS and who have not. Material and Methods. Seventy-nine patients were enrolled in the study. Central venous blood samples were obtained immediately before surgery, during operation, and at the end of surgery to assess TAC. Clinical data regarding patient demographics and operative outcomes were prospectively collected and entered into our clinical database. Results. LCOS developed in 8 patients $(10.12 \%)$. The TAC has decreased sharply in the LCOS patients compared with those who did not develop LCOS $(P<0.001)$ during operation. In addition, the receiver operating characteristic (ROC) area was 0.879 . Conclusion. TAC has decreased during operation in a significant proportion of patients undergoing isolated CABG, and this is more prominent and serious and might be an independent variable in patients who have developed LCOS. This may be related to intraoperative misadventure or inadequate myocardial antioxidative protection. Routine measurement of the TAC during operation may provide information for assessment of the LCOS development.
\end{abstract}

\section{Introduction}

Ischemia-reperfusion injury may cause a damage to the myocardium following blood flow restoration after a critical period of coronary occlusion [1]. In fact, ischemiareperfusion is a clinical problem associated with procedures such as thrombolysis, angioplasty, and coronary bypass surgery which are commonly used to establish the blood reflow and minimize the damage of the heart due to severe myocardial ischemia.

Oxidative stress is a one of the hypotheses to explain the pathogenesis of ischemia-reperfusion injury $[2,3]$. Oxidative stress, which is usually associated with increased formation of reactive oxygen species (ROS), modifies phospholipids and proteins leading to lipid peroxidation and oxidation of thiol groups $[4,5]$. Antioxidant molecules prevent and/or inhibit these harmful reactions [6]. Serum concentrations of different antioxidant molecules can be measured in laboratories separately, but the measurement of these molecules in laboratory is time consuming, labor intensive, and requires complicated techniques. On the contrary, measurement of total antioxidant capacity is easy to use [7]. Advances in myocardial protection have led to lower risk-adjusted rates of morbidity and mortality after coronary artery bypass surgery. Prevalence of postoperative LCOS remains relatively high approximately $9 \%$ [8]. In LCOS it is necessary to have intra-aortic balloon counterpulsation or inotropic support for longer than 30 minutes after the patient was returned to the intensive care unit to maintain the systolic blood pressure higher than $90 \mathrm{~mm} \mathrm{Hg}$ and the cardiac index greater than $2.2 \mathrm{~L} / \mathrm{min}$ per square meter [8]. The current low rates of perioperative infarction and mortality associated with CABG 
TABLE 1: The preoperative characteristics of the patients.

\begin{tabular}{lcc}
\hline & $n$ & Ratio $(\%)$ \\
\hline Male & 60 & 75.94 \\
Female & 19 & 24.06 \\
Age (yr) & $61.5 \pm 9.6$ & \\
Timing & & \\
$\quad$ Elective & 70 & 88.6 \\
$\quad$ Emergency & 9 & 11.4 \\
Recent MI & 11 & 13.92 \\
LVEF & & \\
$\quad 40 \%-60 \%$ & 79 & 100 \\
NYHA class & & \\
$\quad$ I & 5 & 6.3 \\
II & 18 & 22.78 \\
III & 24 & 30.37 \\
IV & 32 & 40.50 \\
CAD & & \\
$\quad$ 1 vessel & 5 & 6.3 \\
2 vessel & 22 & 27.84 \\
3 vessel & 44 & 55.69 \\
Left main CAD & 8 & 10.12 \\
Diabetes & 14 & 17.72 \\
Hypertension & 35 & 44.30 \\
PVD & 6 & 7.59 \\
\hline Abbrevatons & & \\
\hline
\end{tabular}

Abbreviations: LVEF: left ventricular ejection fraction; NYHA: New York Heart Association; CAD: coronary artery disease; PVD: peripheral vascular disease.

have required large sample sizes in order to demonstrate clinically significant differences between myocardial protective strategies [3].

In this paper, we have explored if there is a difference between the coronary artery bypass patients who have developed LCOS and who have not.

According to us this is the first report investigated the changes of total antioxidant capacity in patients who have developed LCOS after CABG [4].

\section{Material and Methods}

Preoperative and operative data were collected prospectively in all patients undergoing CABG between August 2004 and December 2004, at Harran University, Research Hospital. This study was approved by the local ethic committee, and informed consent was obtained from all patients. The preoperative characteristics of the 79 consecutive patients are shown on Table 1.

2.1. Operative Technique. After median sternotomy and full heparinization, cardiopulmonary bypass $(\mathrm{CPB})$ was instituted thorough a single two-stage right atrial cannula and an ascending aortic cannula. During bypass, the hematocrit level, pump flow rates, and mean arterial pressures were maintained between $20 \%$ and $25 \%, 2.0$ and $2.5 \mathrm{~L} / \mathrm{min}$ per square meter, and 50 and $60 \mathrm{~mm} \mathrm{Hg}$ by use of sodium nitroprusside or phenylephrine hydrochloride as required, respectively. In all patients, the heart was arrested by an aortic root infusion of high potassium $(27 \mathrm{mEq} / \mathrm{L})$ blood cardioplegic solution. Distal anastomoses were done under a single cross-clamp. A left internal thoracic artery graft was used in all patients.

2.2. Low Cardiac Output Syndrome. Low cardiac output syndrome was diagnosed in patients who required an intraaortic balloon pump either in the operating room or in the intensive care unit because of hemodynamic compromise or required inotropic medication to maintain the systolic blood pressure greater than $90 \mathrm{~mm} \mathrm{Hg}$ and the cardiac output greater than $\left(\geq 2.5 \mathrm{~L} \cdot \mathrm{min}^{-1} \cdot \mathrm{m}^{-2}\right)$ for at least 30 minutes in the intensive care unit after correction of all electrolytes and blood gas abnormalities while adjusting preload volume to its optimal values [8]. Patients who [5] received dopamine, dobutamine, or epinephrine named inotropic support. Patients who received dopamine lower than $4 \mathrm{mcg} / \mathrm{kg} / \mathrm{min}$ aimed to increase renal perfusion and received vasodilating agents because of a high cardiac output $\left(\geq 2.5 \mathrm{~L} \cdot \mathrm{min}^{-1} \cdot \mathrm{m}^{-2}\right)$ and low peripheral resistance were not considered to have LCOS.

2.3. Samples. Blood samples were drawn from central venous catheter immediately before the anesthetic induction, during operation and at the end of operation. Samples were withdrawn from central vein into heparinized tubes and immediately stored on ice at $4^{\circ} \mathrm{C}$. The plasma was then separated from the cells by centrifugation at $3000 \mathrm{rpm}$ for $10 \mathrm{~min}$, and the plasma samples were stored at $-80^{\circ} \mathrm{C}$ until analysis.

2.4. Biochemical Parameters' Measurement of TAC. The TAC of the plasma was measured using a novel automated colorimetric measurement method developed by Erel [7]. Accurate measurements of TAC can be obtained in as little as $10 \mathrm{~min}$, making this assay eminently suitable for the clinical biochemistry laboratory [9].

2.5. Statistical Analysis. The data analyzed by repeated measurements' variance analysis, Mann Whitney $U$ test, and ROC analysis using SPSS 11.0 and SigmaPlot 8.0 programs [6].

\section{Results}

The patient demographics are listed in Table 1. Table 2 compares the operative data of patients who developed or not LCOS. Complete revascularization was accomplished in $74(93.67 \%)$ patients. The left anterior descending artery (LAD) territory was revascularized in $98.73 \%$ of the patients with LAD disease. Patients who developed LCOS had longer cardiopulmonary bypass time, longer aortic cross-clamp time, longer postoperative intensive care unit stay, increased ventilatory support, and longer hospital stay. TAC was decreased during operation in all patients $(P<0.001)$. It is shown on Table 3 and Figure 1. However, in patients who developed LCOS, TAC presented a sharp initial fall during 
TABLE 2: Operative data of the patients.

\begin{tabular}{lccc}
\hline & Non-LCOS $(n=71)$ & LCOS $(n=8)$ & $P$ \\
\hline Age (yr) & $61.3 \pm 9.6$ & $63.6 \pm 9.6$ & 0.51 \\
Grafts & $2.03 \pm 0.7$ & $1.93 \pm 0.7$ & 0.51 \\
Pump time (min) & $85 \pm 24$ & $110 \pm 39$ & 0.27 \\
Crossclamp time (min) & $60 \pm 18$ & $67 \pm 27$ & 0.83 \\
Days in hospital & $7 \pm 2$ & $13.8 \pm 8.4$ & 0.27 \\
Perioperative myocardial infarction & 0 & $2(25 \%)$ & \\
Mortality & 0 & $1(12.5 \%)$ & \\
\hline
\end{tabular}

TABLE 3: TAC differences in non-LCOS and LCOS patients.

\begin{tabular}{lcccc}
\hline TAC & BO & DO & EO & $P$ \\
\hline Non-LCOS & $1.84 \pm 0.07$ & $1.66 \pm 0.14$ & $1.61 \pm 0.08$ & 0.001 \\
LCOS & $1.82 \pm 0.11$ & $1.52 \pm 0.10$ & $1.30 \pm 0.16$ & 0.002 \\
\hline
\end{tabular}

Values are mean \pm SE.

Abbreviations: BO: before the operation; DO: during the operation; EO: at the end of the operation.

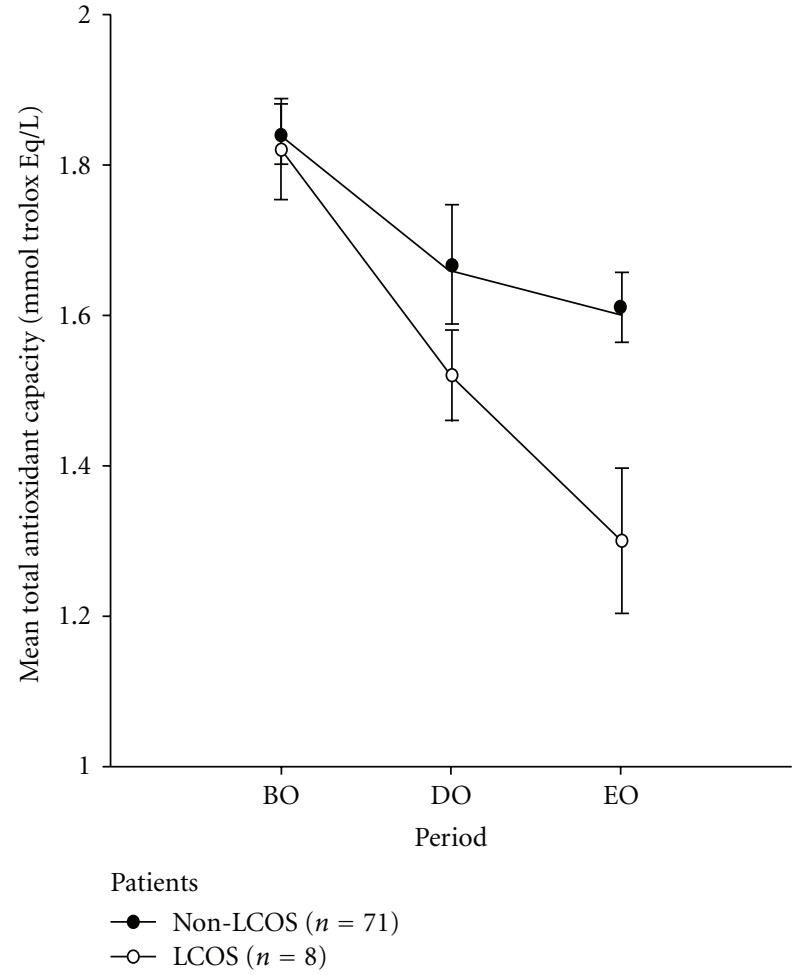

FIgURE 1: Time changes of TAC during coronary artery bypass surgery. All values showed statistically significant difference from baseline values $(P<0.05)$. The values are mean \pm standard error. Abbreviations: $\mathrm{BO}$ : before the operation; DO: during the operation; EO: the end of the operation.

operation $(P<0.001)$. The area of ROC curve was 0.879 as seen on Figure 2, [7].

\section{Discussion}

We found that the patients who are undergoing to CABG are exposed to potent oxidative stress. This situation is

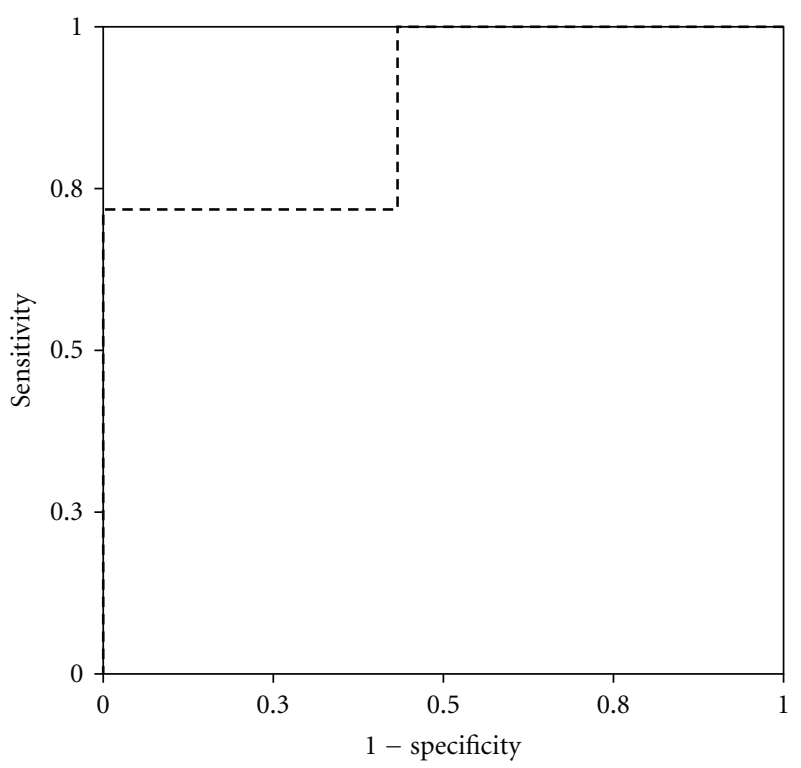

FIGURE 2: ROC curve of the total antioxidant capacity values obtained from at the end of the surgery.

closely connected with overproduction of reactive oxygen species (ROS). ROS is produced during ischemia/reperfusion process and systemic inflammatory response, associated with cardiac surgery performed with CPB $[10,11]$. Preoperative high risk, such as low ejection fraction, recent $\mathrm{MI}$, and renal insufficiency, may lead to inadequate ventricular function in the early postoperative period in patients who developed LCOS undergoing CABG.

In this study LCOS developed in 8 patients (10.12\%). The change of TAC was statistically significant in these patients compared with those who did not develop postoperative LCOS. Although the exact mechanisms and interactions among various antioxidants are not fully understood, it is possible that one antioxidant may equilibrate with another to 
establish a cellular redox potential, and thus all endogenous antioxidants may act in concert to protect against oxidative insult [1]. Several studies have proposed the essential role of ROS in the pathogenesis of myocardial ischemiareperfusion injury $[10,11]$. In ischemic-reperfused hearts, many alterations such as depression in contractile function, arrhythmias, and loss of adrenergic pathways have been observed [12].

The relationship between contractile function and myocardial metabolism is not clear. Thus, alterations in the myocardium during ischemia-reperfusion were suggested to be in part due to oxidative stress. Ischaemia causes alterations in the defence mechanisms against oxygen free radicals. At the same time, production of oxygen free radicals increases. There is evidence of oxidative stress during surgical reperfusion of the whole heart or after thrombolysis, and it is related to transient left ventricular dysfunction or stunning [13]. Our findings in this study support that in other authors' observations, TAC was decreased during 8 operations in all patients $(P<0.001)$ (Figure 1$)$. However, in patients who developed LCOS, TAC presented a sharp fall during and at the end of the operation $(P<0.001)$ (Figure 1$)$.

Various methods of measurement have been developed for total antioxidant status, but there is no accepted reference method yet [7]. In this study, the total antioxidant status of the plasma was measured using a novel automated colorimetric measurement method for the TAC developed by Erel in patients undergoing CABG [6]. We report here that the novel assay has several major advantages over the other techniques currently available. It is rapid (10 min), simple and inexpensive, and easily fully automated. It is also reliable and sensitive and not subject to interference commonly occurring serum components such as bilirubin, serum lipids, and anticoagulants such as heparin or oxalate [6].

In conclusion, during the coronary bypass surgery, oxidants increase, antioxidants decrease, and oxidative stress develops. This is more prominent and more serious in those who developed LCOS. We think that this difference may be related or may be seconder to the etiopathogenesis of the LCOS. In the coronary bypass surgery, we think that antioxidant vitamin supplementation such as vitamin $\mathrm{C}$ and E may be beneficial for these patients. According to our ROC analysis, results of the TAC measurement during the CABG have indicative potential in LCOS development [9].

\section{References}

[1] N. S. Dhalla, A. B. Elmoselhi, T. Hata, and N. Makino, "Status of myocardial antioxidants in ischemia-reperfusion injury," Cardiovascular Research, vol. 47, no. 3, pp. 446-456, 2000.

[2] K. K. Griendling and R. W. Alexander, "Oxidative stress and cardiovascular disease," Circulation, vol. 96, no. 10, pp. 32643265, 1997.

[3] P. Kaplán, J. Lehotský, and P. Račay, "Role of sarcoplasmic reticulum in the contractile dysfunction during myocardial ischaemia and reperfusion," Physiological Research, vol. 46, no. 5, pp. 333-339, 1997.

[4] A. B. Elmoselhi, A. Lukas, P. Ostadal, and N. S. Dhalla, "Preconditioning attenuates ischemia-reperfusion-induced remodeling of $\mathrm{Na}^{+}-\mathrm{K}^{+}$-ATPase in hearts," American Journal of Physiology, vol. 285, no. 3, pp. H1055-H1063, 2003.

[5] S. Suzuki, M. Kaneko, D. C. Chapman, and N. S. Dhalla, "Alterations in cardiac contractile proteins due to oxygen free radicals," Biochimica et Biophysica Acta, vol. 1074, no. 1, pp. 95-100, 1991.

[6] O. Erel, "A novel automated method to measure total antioxidant response against potent free radical reactions," Clinical Biochemistry, vol. 37, no. 2, pp. 112-119, 2004.

[7] O. Erel, "A novel automated direct measurement method for total antioxidant capacity using a new generation, more stable ABTS radical cation," Clinical Biochemistry, vol. 37, no. 4, pp. 277-285, 2004.

[8] V. Rao, J. Ivanov, R. D. Weisel, G. Cohen, M. A. Borger, and D. A. G. Mickle, "Lactate release during reperfusion predicts low cardiac output syndrome after coronary bypass surgery," Annals of Thoracic Surgery, vol. 71, no. 6, pp. 1925-1930, 2001.

[9] M. Harma, M. Harma, and O. Erel, "Measurement of the total antioxidant response in preeclampsia with a novel automated method," European Journal of Obstetrics Gynecology and Reproductive Biology, vol. 118, no. 1, pp. 47-51, 2005.

[10] P. Biglioli, A. Cannata, F. Alamanni et al., "Biological effects of off-pump versus on-pump coronary artery surgery: focus on inflammation, hemostasis and oxidative stress," European Journal of Cardio-Thoracic Surgery, vol. 24, no. 2, pp. 260-269, 2003.

[11] L. Hadjinikolaou, C. Alexiou, A. S. Cohen, R. D. L. Standbridge, A. J. McColl, and W. Richmond, "Early changes in plasma antioxidant and lipid peroxidation levels following coronary artery bypass surgery: a complex response," European Journal of Cardio-thoracic Surgery, vol. 23, no. 6, pp. 969975, 2003.

[12] S. Persad, V. Panagia, and N. S. Dhalla, "Role of $\mathrm{H}_{2} \mathrm{O}_{2}$ in changing $\beta$-adrenoceptor and adenylyl cyclase in ischemiareperfused hearts," Molecular and Cellular Biochemistry, vol. 186, no. 1-2, pp. 99-106, 1998.

[13] R. Ferrari, L. Agnoletti, L. Comini et al., "Oxidative stress during myocardial ischaemia and heart failure," European Heart Journal, vol. 19, pp. B2-B11, 1998. 


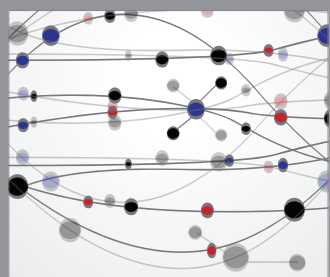

The Scientific World Journal
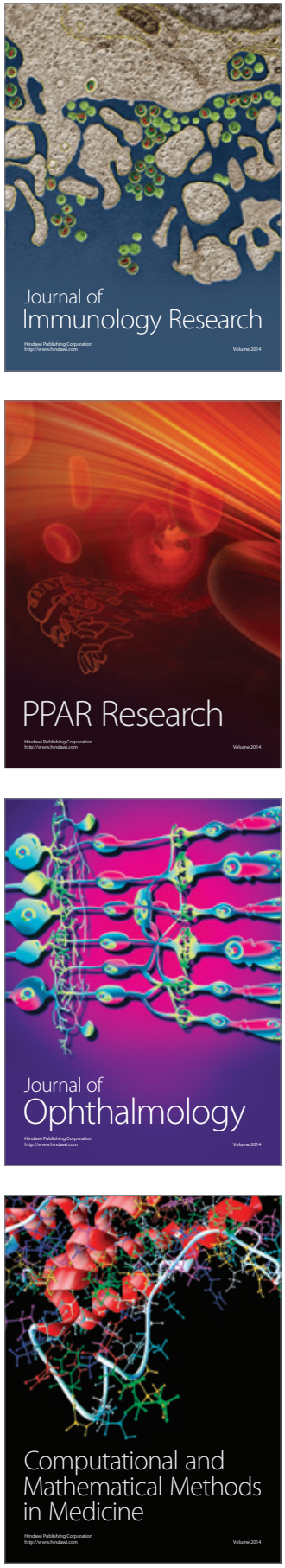

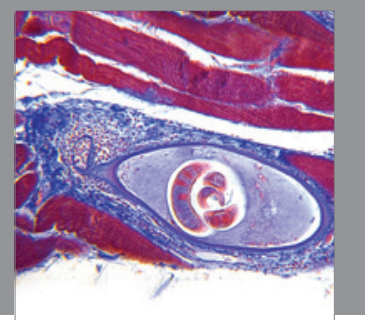

Gastroenterology

Research and Practice
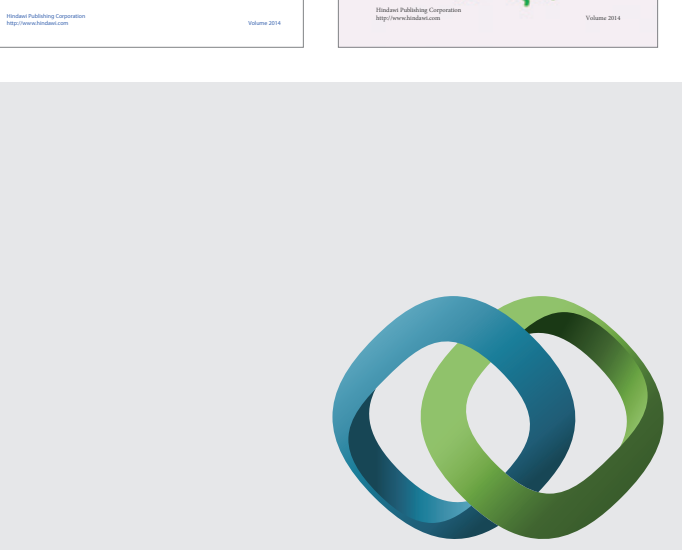

\section{Hindawi}

Submit your manuscripts at

http://www.hindawi.com
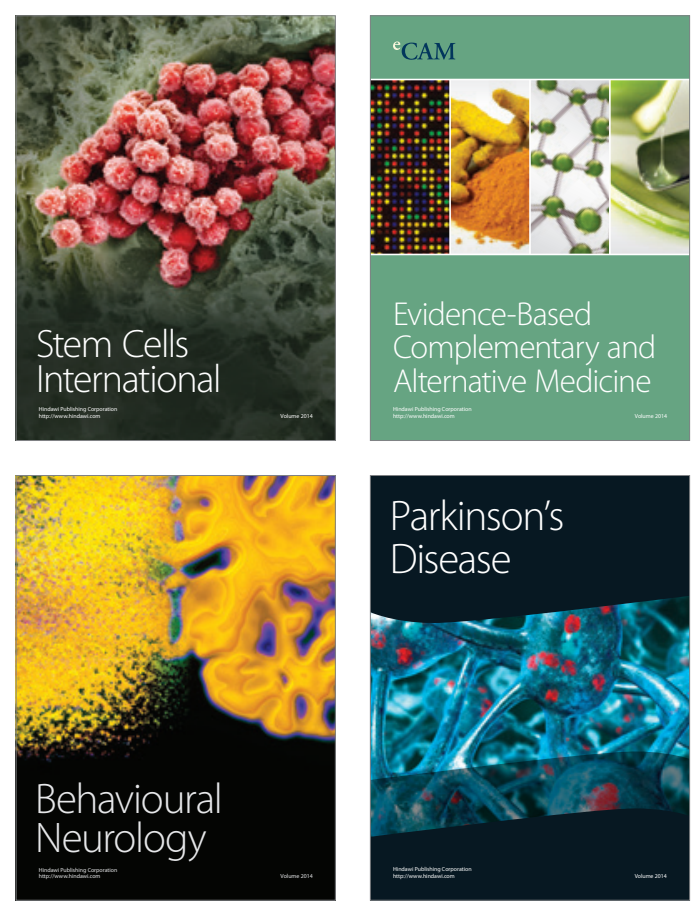

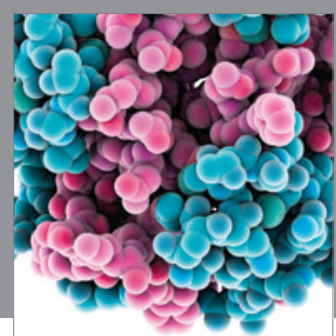

Journal of
Diabetes Research

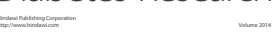

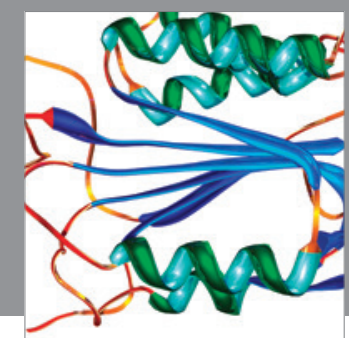

Disease Markers
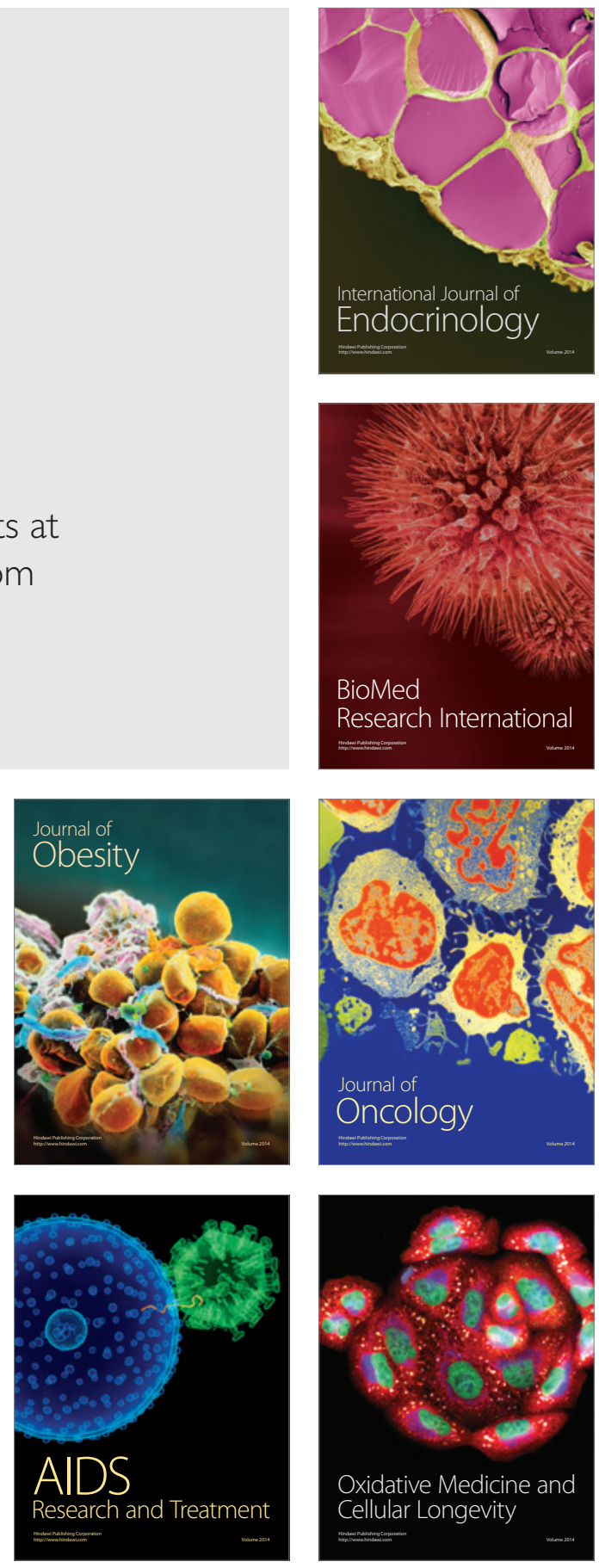